\title{
Conhecimento fonológico em crianças com desenvolvimento típico
}

\section{Phonological knowledge in children with typical development}

Suzana do Couto Mendes

Universidade Federal do Rio de Janeiro, Rio de Janeiro, Rio de Janeiro / Brasil suzanamendes.fono@gmail.com

Christina Abreu Gomes

Universidade Federal do Rio de Janeiro, Rio de Janeiro, Rio de Janeiro / Brasil $\mathrm{CNPq}$

christina-gomes@uol.com.br

Gastão Coelho Gomes

Universidade Federal do Rio de Janeiro, Rio de Janeiro, Rio de Janeiro / Brasil gastao@im.ufrj.br

Resumo: O presente estudo teve como objetivo analisar a emergência da gramática fonológica em crianças com desenvolvimento típico, apoiado na hipótese dos Modelos Baseados no Uso, segundo a qual o conhecimento fonológico concernente às relações fonotáticas e templates lexicais emerge a partir das formas das palavras armazenadas no léxico. Assim, o esperado é que quanto maior o léxico mais estruturas farão parte da gramática fonológica abstraída pelo falante e mais robustez será conferida às representações. Um teste de repetição de não-palavras, com o propósito de avaliar o conhecimento fonológico do falante através da acurácia de repetição, e o teste de vocabulário receptivo Peabody, utilizado para mensurar o tamanho do léxico dos participantes, foram aplicados a 66 crianças com desenvolvimento típico de três faixas etárias diferentes (5, 7 e 9-12 anos). Os resultados encontrados indicaram uma 
relação entre o efeito do tamanho e a frequência dos constituintes das não-palavras, a idade das crianças e do tamanho do léxico e o grau de acurácia de repetição. Escores mais altos de acurácia foram obtidos em itens lexicais com duas e três sílabas, constituídos de sílabas com alta frequência de ocorrência e em crianças com léxico maior, o que ratifica a hipótese de que o conhecimento fonológico se desenvolve em função da aquisição lexical.

Palavras-chave: Modelos Baseados no Uso; conhecimento fonológico; desenvolvimento típico.

Abstract: This study aimed to analyze the emergence of the phonological grammar in children with typical development, based on the Usagebased Models' hypothesis by which phonological knowledge regarding phonotactics and lexical templates emerge from the word forms stored in the lexicon. Hence, it is expected that the higher the lexicon more structures will be part of the speaker's abstracted phonological grammar and robustness will be given to representations. A nonword repetition test, aiming to assess the phonological knowledge of the speaker by the degree of accuracy in repetition, and the receptive vocabulary test Peabody, used to measure the lexicon size of the participants, were applied to 66 children with typical development distributed in three different age groups ( 5 years, 7 years and 9-12 years). The results indicate a relationship between the size and the frequency of the constituents of the nonwords as well as children's age and size of the lexicon and the degree of accuracy in nonword's repetition. Higher scores on accuracy were obtained in lexical items with two and three syllables, made up of high-frequent syllables and by children with a higher lexicon, which ratifies the hypothesis according to which phonological knowledge develops depending on the acquisition of the lexicon.

Keywords: Usage-Based Models; phonological knowledge; typical development.

Recebido em: 22 de março de 2016. Aprovado em: 18 de outubro de 2016. 


\section{Introdução}

O objetivo deste artigo é apresentar evidências que contribuam para identificar o perfil de aquisição típica, relativo ao estabelecimento de representações sonoras abstratas e o gerenciamento dessas representações, a partir de resultados de estudo com crianças com desenvolvimento típico, falantes do português brasileiro, entre 5 e 12 anos (MENDES, 2014), desenvolvido a partir dos pressupostos de modelos fonológicos multirrepresentacionais (BYBEE, 2001; 2010; PIERREHUMBERT, 2003). Estudos têm demonstrado que alguns aspectos do conhecimento fonológico, tomados tacitamente na Linguística como adquiridos quando as crianças vão ser alfabetizadas, ainda não estão plenamente estabelecidos aos 6 anos de idade. Por exemplo, Hazan e Barrett (2000) mostraram que crianças com idades entre 6 e 12 anos ainda não apresentam o mesmo desempenho dos adultos em tarefa de categorização de contraste fonêmico envolvendo diferença de vozeamento e de ponto de articulação de oclusivas e fricativas do inglês, como em /g/-/k/ (goat "bode" - coat "casaco"),/d/-/g/ (date "data" - gate "portão"),/s/-/z/ (sue "processar" - zoo "redução de zoológico"), /s/ - /S/ (sue - shoe "sapato"). Assim, a observação de crianças na faixa etária do grupo estudado pode trazer contribuições para o entendimento do desenvolvimento fonológico.

A hipótese geral que norteou a pesquisa é a de que o conhecimento fonológico é emergente das representações das palavras no léxico, organizadas em redes de conexão lexical por semelhança sonora e semântica, e que conhecimentos de diversos tipos são representados em diferentes níveis de abstração, sendo que cada tipo (ou nível) tem seu próprio aparato representacional. Ainda, os seres humanos são dotados da capacidade de fazer inferência probabilística das representações, sendo que as frequências de tipo de estruturas linguísticas e de ocorrência das palavras têm impacto no acesso e na representação lexical, assim como também na aquisição e mudança linguística (BYBEE, 2010, 2015; PISONI et al., 1985; COLEMAN; PIERREHUMBERT, 1997). Também estudos têm mostrado que os seres humanos são dotados de capacidade de aprendizado estatístico e que essa capacidade se manifesta em crianças com menos de 12 meses de idade (SAFFRAN; ASLIN; NEWPORT, 1996; THIESSEN; SAFFRAN, 2003; PELUCCHI; HAY; SAFFRAN, 2009). Essas postulações são captadas nos Modelos Baseados no Uso, 
também referidos como Modelos Multirrepresentacionais (SILVA; GOMES, 2007).

Existe, portanto, uma relação de dependência entre léxico e desenvolvimento fonológico devido à retroalimentação entre eles. As pesquisas sobre aquisição fonológica apresentam evidências de que, à medida que as crianças ampliam o tamanho do léxico, são produzidos ajustes nas suas produções em relação ao alvo, mas é preciso um conjunto de palavras, observável na fase em que se detecta a produção estável de 50 palavras, ${ }^{1}$ a partir dos 15 meses de idade, para que as produções apresentem generalizações que refletem moldes ou templates lexicais de sua língua. Essas produções se distanciam das formas das palavras no input, embora apresentem semelhanças entre si, e são interpretadas como indicativas de que as crianças estão fazendo generalizações e estabelecendo representações de categorias fonológicas presentes nas palavras adquiridas (VELLEMAN; VIHMAN, 2006; VIHMAN, 2009; 2014). Nesta fase, novas palavras adquiridas são enquadradas nos templates estabelecidos pelas crianças. Em outras palavras, a estruturação do componente fonológico se dá através de generalizações sobre a forma das palavras representadas no léxico e a crescente estruturação fonológica permite a aquisição de novas palavras.

Partindo das hipóteses mencionadas nos parágrafos anteriores, foi observado o comportamento de crianças com desenvolvimento típico na repetição de não-palavras, ${ }^{2}$ estímulos formados por segmentos existentes no português brasileiro em estrutura silábica comum na língua, do tipo Consoante Vogal - CV, considerando três templates ou moldes lexicais, de 2, 3 e 4 sílabas e diferentes frequências de tipo, isto é, sílabas de alta e baixa frequência de tipo. $O$ teste de repetição de não-palavras fornece informação sobre as generalizações e representações estabelecidas pelo falante, isto é, sobre o conhecimento fonológico que é utilizado

${ }^{1} \mathrm{O}$ ponto de 50 palavras não significa que este é o número mínimo exato requerido, mas que metodologicamente é o ponto utilizado para se verificar a forma das produções das crianças.

${ }^{2} \mathrm{O}$ termo não-palavra tanto é usado para se referir a estímulos compostos por segmentos possíveis na língua e de acordo com as relações fonotáticas e estruturas silábicas presentes na língua, mas que não constituem palavra do léxico, como também para estímulos formados por sequências e segmentos inexistentes. $\mathrm{O}$ primeiro tipo de estímulo também é referido como pseudopalavra (FRISCH; LARGE; PISONI, 2000). Neste trabalho optou-se pelo uso do termo não-palavra. 
para repetir as não-palavras. Assim, um baixo desempenho neste tipo de repetição pode indicar que determinadas representações não são suficientemente robustas. Por outro lado, torna-se importante verificar o tamanho do léxico dos sujeitos estudados, pois um léxico menor do que o esperado para determinada idade pode ser preditivo do desempenho em uma tarefa de repetição. Considerando que as representações em diversos níveis de abstração são emergentes das representações das palavras no léxico, as crianças tiveram o tamanho do léxico aferido pelo teste Peabody (DUNN; DUNN, 1997). Assim, o objetivo específico é o de observar a organização e estruturação do conhecimento fonológico em crianças com desenvolvimento típico, usando a medida de acurácia de repetição de não-palavras em crianças falantes do português brasileiro.

$\mathrm{O}$ artigo está organizado da seguinte maneira: a seção 2 trata das principais questões teóricas postuladas no âmbito dos Modelos Baseados no Uso no que diz respeito à organização do conhecimento fonológico dos falantes; na seção 3, são apresentadas as hipóteses de trabalho e a metodologia utilizada na obtenção dos dados, seleção dos sujeitos, bem como os testes utilizados e variáveis de análise; na seção 4, são apresentados os resultados obtidos e a análise desses resultados à luz de evidências encontradas na literatura; e, finalmente, são apresentadas as considerações finais.

\section{Sobre a emergência do conhecimento fonológico e sua organização}

De acordo com os Modelos Baseados no Uso, a gramática ou conhecimento linguístico emerge da relação entre aspectos cognitivos inatos e a experiência do falante com o input. A postulação básica é que não apresentamos um aparato especificamente linguístico inato, mas que os seres humanos são dotados de um aparato cognitivo geral inato que permite extrair padrões de informação presentes no ambiente, fazer generalizações, analogias e categorizações para organizar e estabelecer sistemas cognitivos, dentre eles a linguagem humana. Assim, a gramática vai sendo estabelecida através do uso da linguagem, através da experiência do falante em ouvir e produzir enunciados em contextos interacionais e sociais e o aparato cognitivo geral (BYBEE, 2010). Mais recentemente, o conceito de sistemas dinâmicos ou sistema adaptativo complexo (BECKNER et al., 2009; BYBEE, 2015) também tem sido 
utilizado para caracterizar a linguagem humana e se mostra bastante adequado para acomodar os postulados dos Modelos Baseados no Uso.

Quanto à organização e estruturação do sistema fonológico, postula-se uma organização na qual as representações fonológicas são múltiplas, pois englobam diferentes tipos, organizados em diferentes graus de abstração, que vão desde o conhecimento de aspectos acústicos perceptuais e de características articulatórias dos sons da fala, até o conhecimento de nível mais abstrato, como unidades que compõem a forma das palavras, como sílabas e segmentos, relações fonotáticas, isto é, de possibilidades de combinações dos sons, incluindo também o conhecimento da indexação social expressa nas formas sonoras, isto é, o conhecimento da variação sociolinguística (PIEREHHUMBERT, 1999; MUNSON; EDWARDS; BECKMAN, 2005; DOCHERTY; FOULKES, 2000, 2014), sendo os níveis mais abstratos emergentes das representações das palavras no léxico.

$\mathrm{Na}$ proposta de Pierrehumbert (2003), são estabelecidos vários tipos (ou níveis) de conhecimento sonoro e graus de abstração, sendo estes organizados probabilisticamente da seguinte maneira: a) Fonética paramétrica - contém a representação dos gestos articulatórios necessários para a produção e também a representação perceptual do sinal acústico em diferentes situações; b) Codificação fonética - representa o inventário fonético da língua para codificação da forma das palavras, em que unidades são abstraídas a partir do espaço fonético paramétrico. Estas unidades contêm informação redundante e devem ser entendidas como picos de áreas que a língua explora preferencialmente. Neste nível também se incluem a prosódia e a entonação; c) Forma das palavras no léxico - envolve a representação da estrutura sonora das palavras no léxico, que permite que cada palavra seja reconhecida apesar de sua variabilidade. Segundo Pierrehumbert (2012, p. 173-174), as informações sonoras das entradas lexicais são de dois tipos: abstratas e detalhadas, isto é, também incluem informações sobre detalhes fonéticos e propriedades sócio-indexais; d) Gramática Fonológica - consiste na representação dos padrões fonológicos mais abstratos, como sílaba, moldes lexicais, incluindo a estruturação prosódica e as restrições fonotáticas da língua; e) Correspondências Morfofonológicas - considerando que muitas alternâncias morfofonológicas não podem ser estabelecidas em função de padrões fonológicos, este nível trata de relações específicas entre 
palavras e paradigmas, generalizações entre pares e conjuntos de itens lexicais, emergência e produtividade de morfemas.

Em Munson, Edwards e Beckman (2005), seguindo a mesma linha de postulados dos Modelos Baseados no Uso, os autores consideram que o conhecimento fonológico é formado por quatro tipos: o conhecimento acústico e perceptual (perceptual knowledge) e o conhecimento articulatório (articulatory knowledge) das características dos sons da fala; o conhecimento de nível mais abstrato, envolvendo a maneira como as palavras são estruturadas e as restrições fonotáticas ( higher level phonological knowledge); e o conhecimento da variabilidade da pronúncia indexada socialmente (social-indexical knowledge).

Em ambas as propostas, a modelagem do conhecimento fonológico engloba aspectos que têm sido excluídos, isto é, não contemplados, nos modelos clássicos da Teoria Fonológica, especificamente o detalhe fonético (de propriedades acústicas e articulatórias, conjugando percepção e produção) e a variabilidade estudada sob o rótulo da Sociolinguística Variacionista. Outro aspecto importante é o fato de que as representações mais abstratas são emergentes de níveis ou tipos de conhecimento menos abstratos, ou seja, a organização e estruturação do sistema fonológico ocorrem no sentido de baixo para cima (bottom-up), quer dizer, as representações de características fonéticas constituem a base para o estabelecimento de representações mais abstratas, como, por exemplo, os templates ou moldes lexicais e as relações fonotáticas.

A representação do detalhe fonético é captada através da modelagem da Teoria de Exemplares (TE). A TE é um modelo de categorização e percepção (HINTZMAN, 1986; NOSOFVSKI, 1986), inicialmente proposto para o estudo da visão, e que foi trazido para a Linguística por Johnson (1997). De acordo com essa proposta, as representações mentais são episódicas, no sentido de que englobam detalhes do objeto da experiência. Em relação às representações linguísticas, estas se caracterizam por incluir informações acústicas e articulatórias detalhadas que se baseiam na experiência do falante com o uso linguístico (PIERREHUMBERT, 2001).

Também para este modelo, o léxico se organiza multidimensionalmente, não como uma lista, como propõem os modelos clássicos, mas sim em redes de conexões através de semelhanças sonoras, semânticas e sonoras e semânticas entre as palavras (BYBEE, 1995). Essa postulação foi primeiramente estabelecida por Pisoni et al. (1985). Os autores 
descrevem resultados de três projetos relacionados ao reconhecimento de palavra falada e à estrutura do léxico. Foram realizados três experimentos de priming auditivo. No primeiro experimento, os estímulos variavam em relação ao grau de semelhança entre prime e alvo - compartilhamento de $0,1,2,3$ ou 4 segmentos iniciais de palavras do inglês, sendo o alvo apresentado com ruído e o prime sem ruído. Os resultados indicaram que, quanto maior o compartilhamento de semelhanças, melhor a identificação do estímulo. Um segundo experimento foi conduzido contendo primes formados por pseudopalavras e foi observado o mesmo resultado obtido para o primeiro experimento. $\mathrm{O}$ mesmo resultado também foi obtido para o terceiro experimento que continha estímulos com compartilhamento de semelhanças no final das palavras. Esses resultados indicaram que o desempenho no reconhecimento de palavras é uma função do grau de semelhança entre prime e alvo, o que, para os autores, indicou que a ativação de uma palavra também ativa outras formas foneticamente semelhantes no reconhecimento lexical. Também foi observada a organização estrutural das palavras no léxico, através do grau de semelhança entre as palavras no léxico, e estabelecendo-se a densidade de vizinhança entre elas. Os autores propuseram que as palavras são representadas no léxico em um espaço fonético-acústico de maneira que as formas mais semelhantes estão mais próximas entre si, o que implica dizer que as palavras estão representadas no léxico em função de relações de semelhança. Essa proposta foi incorporada nos modelos aqui apresentados.

Há evidências relativas à emergência do conhecimento fonológico em crianças com desenvolvimento típico que mostram a relação estreita entre conhecimento fonológico e aquisição lexical. Ferguson e Farwell (1975) constataram que a aquisição de sons e estruturas fonológicas pelas crianças se dá em função das palavras que vão adquirindo e não independentemente. As evidências encontradas indicaram que a crianças podem produzir de maneiras diferentes o mesmo som presente em palavras-alvo diferentes, o que sugere que a representação da criança está pautada nas informações sobre os itens lexicais e não nas características abstratas dos sons que as compõem. Os autores também observaram diferenças individuais fortes nas preferências das crianças em relação a estratégias e itens lexicais. Além disso, Vihman e Kunnari (2006), comparando crianças adquirindo inglês, francês e finlandês, identificaram aspectos da emergência do conhecimento fonológico 
à medida que o léxico da criança aumenta. Segundo as autoras, no ponto em que as crianças produzem 4 palavras consistentemente, em torno dos 10 e 12 meses, fase das primeiras palavras, as produções se caracterizam por não apresentar uma coerência interna, no sentido de que não é possível observar um padrão de produção utilizado pelas crianças, e normalmente a forma sonora das palavras contém padrões motores (sons) encontrados na fase final do balbucio e também presentes na forma sonora da palavra-alvo. Quando as produções atingem um conjunto em torno de 25 itens lexicais diferentes, fase das formas selecionadas, há mais semelhanças entre a forma produzida pela criança e a forma do adulto, e a criança parece selecionar determinadas palavras para tentar produzi-las como os adultos. Com o aumento do tamanho do léxico, em torno de 50 palavras, as crianças fazem inferência sobre os itens lexicais representados e estabelecem generalizações, fase das palavras adaptadas. Nesta fase, as palavras são mais parecidas entre si e se distanciam da forma alvo, sendo sua forma menos acurada que a fase anterior. Essas produções são tomadas como manifestações de estabelecimento de padrões fonológicos abstratos (templates) que a criança atribui a outros itens que vão sendo adquiridos. Os templates são identificáveis quando se observa sistematicidade de forma para um conjunto de itens que passam a compartilhar características sonoras e estruturais, apresentando uma coerência interna, e se distanciando do alvo adulto. À medida que a aquisição lexical prossegue, novos esquemas motores vão sendo automatizados e representados, novas generalizações vão sendo estabelecidas, e, assim, a criança vai organizando os diversos tipos de conhecimento fonológico na direção da língua alvo.

Vihman e Croft (2007) propõem que a organização fonológica se baseia em templates, isto é, moldes lexicais definidos em função da estrutura silábica. Durante a aquisição, os templates são o resultado do estabelecimento de generalizações feitas pelas crianças, tendo como base os padrões fonéticos das primeiras produções/representações, e não são inatos, porque diferem entre crianças adquirindo a mesma língua e entre crianças adquirindo línguas diferentes. Na proposta de Vihman e Croft, o aspecto da estrutura segmental das palavras se resolve em função de moldes fonotáticos que são específicos de cada língua. $\mathrm{O}$ modelo não inclui estruturas inatas e a continuidade entre crianças e adultos reside na hipótese de que o desenvolvimento do conhecimento sobre a estrutura linguística é gradual. Ainda, embora nem o formato e nem a quantidade 
das representações das crianças sejam as mesmas dos adultos, o processo de diferenciar e generalizar o conhecimento sobre as formas das palavras vai levar ao modelo de representação baseado em templates dos adultos.

Também têm sido encontradas evidências que apontam a importância de se considerar a representação do detalhe fonético como parte do conhecimento linguístico do falante (GOLDINGER, 1996; PIERREHUMBERT, 1999; CLOPER; CONREY; PISONI, 2005), assim como a representação de unidades sublexicais mais abstratas (PISONI et al., 1985; VITEVITCH; LUCE, 1999).

Sobre a independência entre diferentes níveis de representação, por exemplo, Beckman, Munson e Edwards (2007) demonstraram, através de um estudo com duas populações clínicas, que, ao menos, dois desses níveis podem funcionar de forma independente. Foram avaliados dois grupos clínicos formados por crianças com Distúrbio Específico da Linguagem (DEL) e com Distúrbio Fonológico (DF) e dois grupos de crianças com desenvolvimento típico (DT), um com idade inferior à das crianças dos grupos clínicos e outro pareado em relação à idade. As crianças foram submetidas a uma tarefa de repetição de não-palavras formadas exclusivamente por difones de alta frequência e de não-palavras formadas por difones de baixa frequência no inglês. No grupo clínico com DEL com idades entre 8 e 13 anos, os resultados obtidos mostraram um desempenho baixo das crianças em relação aos dois tipos de estímulos, sendo o desempenho pior em relação aos estímulos com constituintes de baixa frequência de tipo. Além disso, embora o desempenho esteja correlacionado à idade e tamanho do léxico, o tamanho do léxico se mostrou o melhor preditor do desempenho das crianças, ou seja, quanto maior o léxico, melhor o desempenho no teste e menor a diferença de desempenho entre os estímulos. Tanto os escores de tamanho de léxico quanto de desempenho no teste para o grupo controle foram superiores aos do grupo DEL. Os resultados encontrados foram interpretados como indicadores de que as crianças com DEL têm dificuldade em fazer representações mais abstratas a partir das representações episódicas ou detalhadas dos itens lexicais estocados no léxico e, por isso, têm desempenho pior do que as crianças controle de mesma idade, pois apresentam dificuldade para atribuir a representação do segmento correto a sequências não familiares ou inexistentes na tarefa de repetição de nãopalavras. Como há uma relação de retroalimentação entre representações e abstrações, as crianças com DEL têm dificuldade de adquirir novos 
itens lexicais e ajustar as representações desses itens devido ao déficit representacional. Por outro lado, a dificuldade de adquirir novos itens impede a expansão do conhecimento fonológico, isto é, tem consequência nos tipos de abstrações que essas crianças podem fazer. Assim, os autores situaram o déficit das crianças com DEL no nível da gramática fonológica de Pierrehumbert (2003) apresentado anteriormente nesta seção. Com relação às crianças com distúrbio fonológico (DF) com idades entre 3 e 6 anos, foi observado um comportamento semelhante destas ao das crianças com desenvolvimento típico de mesma idade no teste de repetição de não-palavras. Por outro lado, as crianças com DF mostraram discrepância em relação às de mesma idade do grupo controle quanto às articulações não apropriadas para a idade e, neste caso, mostraram um desempenho semelhante ao das crianças mais novas. Esses resultados sugerem que, neste grupo clínico, o nível de representação alterado é o nível de codificação perceptual do sinal acústico da fala. $\mathrm{O}$ fato de elas não apresentarem comportamento diferente ao das crianças de mesma idade no processamento dos estímulos foi tomado como indicativo de que as crianças com DF estabelecem abstrações a partir das representações no léxico, da mesma maneira que as crianças com desenvolvimento típico. Os autores concluem que crianças com DF são capazes de estabelecer representações mais abstratas ao mesmo tempo em que apresentam dificuldades em estabelecer representações acuradas do detalhe fonético. Para os autores, esses resultados, tomados em conjunto, fornecem evidência para a proposta de Pierrehumbert (2003) de representação do conhecimento fonológico em diferentes níveis de abstração, além de situar os dois tipos de déficit em relação a esses níveis.

O efeito de frequência de tipo dos constituintes das não-palavras do trabalho de Beckman et al. (2007) também fornece evidência sobre a inferência probabilística de categorias fonológicas a partir das representações das palavras no léxico. Em trabalho anterior, Frish, Large e Pisoni (2000) também mostraram que o processamento de não-palavras por falantes adultos envolve inferência probabilística sobre o conjunto de itens que faz parte do léxico. Foi constatado efeito de frequência de tipo de constituintes de não-palavras em duas tarefas diferentes: julgamento de aceitabilidade da não-palavra, considerando seu grau de semelhança a uma palavra real do inglês, e reconhecimento do estímulo. Os falantes do inglês avaliaram como mais parecidas com as palavras da língua aqueles estímulos formados por constituintes de 
alta probabilidade na língua, assim como estes estímulos foram mais lembrados na tarefa de memória de reconhecimento. Segundo os autores, os resultados encontrados indicam que os padrões lexicais presentes nas representações das palavras no léxico constituem a base para a emergência de uma competência fonológica que é colocada em uso em tarefas de processamento de não-palavras.

Na seção a seguir, são apresentadas as hipóteses específicas de trabalho bem como a metodologia utilizada para o desenvolvimento da pesquisa.

\section{Método}

A hipótese de trabalho é a de que o conhecimento fonológico relacionado à estrutura das palavras (templates) e das relações fonotáticas entre os constituintes das sílabas emerge das representações das palavras armazenadas no léxico. Assim, em uma tarefa de repetição, que avalia a acurácia com que a criança repete estímulos formados por sequências possíveis na língua, mas que não constituem palavras reais, espera-se que as crianças com mais idade e, consequentemente, com um léxico maior, apresentem um melhor desempenho no teste de repetição de não-palavras que as crianças com menos idade e um léxico menor, porque apresentam um léxico de tamanho suficiente para conferir robustez às diversas relações fonotáticas e moldes lexicais mapeados nos estímulos do teste. Conforme mencionado na seção anterior, Beckman, Munson e Edwards (2007) mostraram tanto correlação entre acurácia de repetição e idade quanto entre acurácia e tamanho do léxico, embora os autores tenham mostrado que estatisticamente o tamanho do léxico seja o melhor preditor do comportamento das crianças por eles estudadas. O esperado é que o léxico das crianças mais velhas seja maior que o das crianças mais novas, mas não necessariamente crianças de mesma idade têm o mesmo tamanho de léxico. Assim, essas variáveis constituem medidas independentes. A relação entre tamanho do léxico e crescente estruturação fonológica, também adotada por Beckman, Munson e Edwards (2007), decorre da hipótese citada na seção anterior da emergência de representações abstratas, gramática fonológica, a partir das representações das palavras no léxico (PIERREHUMBERT, 2003). Um teste que avalia a acurácia de repetição de não-palavras acessa a capacidade das crianças em estabelecer representações abstratas relacionadas a moldes lexicais e relações 
fonotáticas, um dos tipos de conhecimento fonológico mencionados em Pierrehumbert (2003) e Munson, Edward e Beckman (2005).

Para verificar essa hipótese, foi utilizado o teste elaborado por Esteves $(2013)^{3}$ com a finalidade de verificar a acurácia das crianças em função do tamanho do léxico e da idade das crianças, e de diferentes templates ou moldes lexicais de palavras do português brasileiro, definidos em função do número de sílabas e da frequência fonotática dos componentes da sílaba, todas do tipo CV (Consoante Vogal). Conforme já mencionado anteriormente, existe uma relação de dependência entre léxico e desenvolvimento fonológico, o que significa dizer que a emergência do conhecimento fonológico se dá através de generalizações sobre a forma das palavras no léxico, quanto mais itens lexicais adquiridos, mais abstrações são estabelecidas, ao mesmo tempo em que a crescente estruturação fonológica permite a aquisição de novas palavras. $\mathrm{O}$ desempenho em um teste de repetição de não-palavras fornece informação a respeito das abstrações estabelecidas a partir do léxico, pois o indivíduo utiliza essas informações para repetir as não-palavras, uma vez que não há acesso direto à uma representação lexical. Por outro lado, dificuldades neste tipo de repetição podem indicar que determinadas representações não são robustas, e essas dificuldades podem ser devidas a um léxico reduzido para a idade, tendo como consequência alguma alteração no conhecimento fonológico e na aquisição de novas palavras..

Diversos trabalhos (GATHERCOLE, 1995; MUNSON; KURTZ; WINDSOR, 2005; BECKMAN; MUNSON; EDWARDS, 2007) têm demonstrado que a acurácia na repetição de não-palavras está diretamente relacionada ao tamanho do léxico. Gathercole (1995) argumenta que a repetição de não-palavras formadas por sequências fonotáticas de baixa frequência na língua também envolveria habilidades de memória de trabalho, armazenamento de informação de curto prazo, devido a sua forma fonológica desconhecida, e que a repetição de estímulos com sequências de alta frequência dependeria do léxico. Assim sendo, as crianças, e também os adultos, utilizariam seus conhecimentos lexicais para recuperar itens do léxico que são parecidos com a estrutura fonológica de determinada não-palavra, estímulos com constituinte de alta frequência no léxico, sendo importante, portanto, o tamanho

\footnotetext{
${ }^{3}$ Em Mendes (2014), foram aplicados ainda o teste ABFW, parte fonológica, a avaliação simplificada do Processamento Auditivo Central e o teste Dicótico de Dígitos.
} 
do léxico. Já a memória de trabalho seria utilizada para a repetição de estímulos com constituintes de baixa frequência. Para Munson et al. (2005), a acurácia de repetição independe da memória de trabalho. Esses autores enfatizam que a acurácia na repetição de não-palavras, compostas tanto por sequências fonotáticas de alta como de baixa frequência, é fortemente influenciada pelo tamanho do léxico do falante. Por outro lado, segundo Pierrehumbert (2003), o léxico de um adulto é de tamanho suficiente para conferir robustez a qualquer representação, inclusive para sequências fonotáticas de baixa probabilidade de ocorrência, e, portanto, não seria esperado efeito de frequência entre adultos. Assim, uma questão que se coloca diz respeito ao momento do desenvolvimento em que as estruturas avaliadas através dos estímulos do teste de repetição de nãopalavras constituem representações robustas.

O teste de repetição de não-palavras contém 30 estímulos com sílaba CV que diferem em número de sílabas (2, 3 e 4 sílabas) e frequência fonotática entre consoante e vogal (sílabas de alta ou de baixa frequência na língua). ${ }^{4}$ As não-palavras são todas com acentuação paroxítona, que constitui o padrão acentual mais frequente na língua para os três tamanhos de acordo com o levantamento feito na base ASPA/UFMG para frequência de tamanho de item lexical, padrão acentual e para a frequência de sílabas CV em todas as posições prosódicas dos três tamanhos utilizados no teste (GOMES et al., 2015). São 10 não-palavras de duas sílabas, 10 de três sílabas e 10 de quatro sílabas. Metade dos estímulos de cada tamanho é composto somente de sílabas de alta frequência no léxico (frequência de tipo) e a outra metade é composta somente de sílabas de baixa frequência. Os estímulos com sílabas compostas de relações fonotáticas de baixa frequência podem incluir relações não atestadas na língua, como no experimento de Coleman e Pierrehumbert (1997). A lista das não-palavras encontra-se no Quadro 1, a seguir.

\footnotetext{
${ }^{4} \mathrm{O}$ teste de não-palavras foi elaborado dentro do projeto "Gramática emergente e inferência estatística no léxico”, coordenado pela Profa. Christina Abreu Gomes no PPGL/UFRJ.
} 


\section{QUADRO 1}

Lista de estímulos utilizados no teste de repetição de não-palavras função do tamanho e da frequência de tipo dos constituintes da sílaba

\begin{tabular}{|c|c|c|c|}
\hline & Duas sílabas & Três sílabas & Quatro sílabas \\
\hline $\begin{array}{c}\text { Menos } \\
\text { freq. }\end{array}$ & $\begin{array}{l}\text { gofu } \\
\text { zefə } \\
\text { JeאU } \\
\text { noאI } \\
\text { zubi }\end{array}$ & 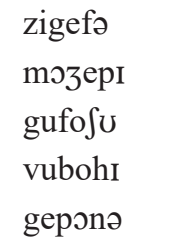 & 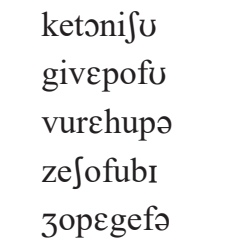 \\
\hline $\begin{array}{l}\text { Mais } \\
\text { freq. }\end{array}$ & $\begin{array}{l}\text { t } \text { ibə } \\
\text { had3I } \\
\text { sebə } \\
\text { ması } \\
\text { mibə }\end{array}$ & $\begin{array}{l}\text { kopizə } \\
\text { tareku } \\
\text { mesivə } \\
\text { vabitu } \\
\text { detukə }\end{array}$ & $\begin{array}{l}\text { helanitu } \\
\text { kovitunə } \\
\text { dzimerotə } \\
\text { mekulivə } \\
\text { moliratu }\end{array}$ \\
\hline
\end{tabular}

As não-palavras foram gravadas por voz feminina de idade em torno de 25 anos e apresentadas para cada criança por meio de computador em ordem aleatória. O programa utilizado para a execução do teste foi o software DMDX (FORSTER, 2003) para ambiente Windows. Os participantes utilizaram um fone de ouvido com microfone acoplado da marca Philips modelo SHMI 900 - stereo pc headset. Na aplicação do teste, as crianças foram instruídas a ouvir o estímulo e em seguida repeti-lo. A aplicação do teste também foi gravada na íntegra em aparelho digital SONY NWD-B103F. A transcrição das respostas foi realizada por duas pessoas e as interpretações discrepantes checadas novamente..

Além do teste de repetição de não-palavras, foi aplicado também o teste do vocabulário receptivo Peabody III (DUNN; DUNN, 1997). O teste Peabody III é um teste de compreensão administrado individualmente que tem como finalidade quantificar o tamanho do léxico de crianças (a partir de 2 anos e meio) e adultos. Consiste de várias pranchas organizadas em grau crescente de dificuldade, cada uma contendo 4 figuras distintas, totalizando 204 vocábulos. É solicitado ao avaliado que identifique, apontando entre as quatro figuras, aquela que corresponde ao significado da palavra enunciada pelo examinador. O teste Peabody está sendo padronizado para o Português brasileiro (CAPOVILLA; 
CAPOVILLA, 1997) e tem sido utilizado em trabalhos com população infantil (FERRACINI et al., 2006; ARAÚJO; MARTELETO; SCHOENFERREIRA, 2010; ESTEVES, 2013).

Os voluntários desta pesquisa são 66 crianças com desenvolvimento típico distribuídas em três diferentes grupos de acordo com a faixa etária: Grupo 1 (G1), 20 crianças com faixa etária de 5 anos e que se encontravam no período pré-escolar, antiga educação infantil; Grupo $2(\mathrm{G} 2)$, composto por 23 crianças com faixa etária de 7 anos e que se encontravam no início de período escolar, $2^{\circ}$ ano do ensino fundamental; Grupo 3 (G3), composto por 23 crianças com faixa etária entre 9 e 12 anos e que se encontravam no $4^{\circ}$ e $5^{\circ}$ anos do ensino fundamental. As crianças, em sua maioria, são alunos da Escola Municipal Desembargador Montenegro, do município do Rio de Janeiro, sendo apenas oito participantes de escolas particulares variadas, escolhidos de acordo com os mesmos critérios de faixa etária e nível de escolaridade. As faixas etárias foram definidas considerando o universo abarcado no trabalho de Hazan e Barrett (2000). Conforme mencionado na Introdução, a literatura tradicional assume que o conhecimento fonológico já está plenamente estabelecido em torno dos 6 anos de idade, mas as evidências empíricas não corroboram esta afirmação e, assim, a observação desses grupos etários pode trazer evidências empíricas que contribuam para essa discussão.

Para a seleção dos participantes, foram utilizados os seguintes critérios de inclusão: ter idade dentro da faixa etária prevista no estudo (G1- 5 anos; G2 - 7 anos; G3 - 9 a 12 anos); não ter queixas ou histórico de quaisquer desordens significativas de aprendizagem, linguagem e/ou audição; não ter repetência escolar.

Os participantes selecionados de acordo com os critérios descritos anteriormente foram então submetidos ao teste de repetição de nãopalavras e ao teste do vocabulário receptivo Peabody III. Os testes foram realizados após os responsáveis terem sido informados sobre os mesmos e após lerem e assinarem o termo de consentimento livre e esclarecido, conforme a orientação aprovada pelo Comitê de Ética em Pesquisa do Instituto de Neurologia Deolindo Couto da Universidade Federal do Rio de Janeiro sob o número de parecer 112.281. 


\section{Resultados}

Os resultados apresentados a seguir se baseiam na medida de acurácia estabelecida em função das correspondências entre os segmentos produzidos pela criança e os segmentos do estímulo-alvo. Foram atribuídos 2 pontos para o segmento igual ao do estímulo, 1 ponto para o segmento substituído, - 1 ponto para o segmento acrescido e 0 ponto no caso de omissão. Os valores foram somados e divididos pelo número de segmentos do estímulo e, assim, foram obtidos escores entre 0 e 1 para cada estímulo repetido do teste (cf. ESTEVES, 2013). O critério de pontuação parte do padrão proposto por Ingram (2002). No entanto a referida proposta ignora as situações em que há acréscimo de segmentos, o que foi resolvido na proposta de Esteves (2013), evitando-se que produções com acréscimo de segmento recebam a mesma pontuação de produções iguais ao alvo, um dos problemas da proposta de Ingram.

Os escores obtidos foram submetidos a um teste de normalidade, para verificar se se encontravam em distribuição normal, isto é, se havia variabilidade em torno dos valores obtidos e se estes se concentram em torno de uma média. Para a análise estatística foi utilizado o teste Kruskall-Wallis, um teste não-paramétrico para variáveis contínuas, uma vez que os escores de acurácia não apresentaram distribuição normal.

A Tabela 1, a seguir, apresenta o comportamento das crianças nas três faixas etárias em função de medidas descritivas dos escores obtidos, considerando o percentual de repetições iguais ao estímulo, isto é, com escore igual a 1 , o percentual de repetições com escore abaixo de $0,7 \mathrm{e}$ a média de escore por grupo etário.

\section{TABELA 1}

Medidas descritivas dos escores de repetição de não-palavras por grupo etário

\begin{tabular}{cccc}
\hline Grupo Etário & $\begin{array}{r}\text { Respostas Iguais } \\
\text { ao alvo (\%) } \\
\text { Escore }=1\end{array}$ & $\begin{array}{c}\text { Respostas com escore } \\
\text { abaixo de 0,7 (\%) }\end{array}$ & $\begin{array}{c}\text { Média dos } \\
\text { escores }\end{array}$ \\
G1 & $51,6 \%$ & $4,5 \%$ & 0,93 \\
G2 & $59,9 \%$ & $4,1 \%$ & 0,94 \\
G3 & $72,5 \%$ & $1,5 \%$ & 0,96 \\
\hline
\end{tabular}

kruskal-wallis chi-squared $=43.7271, \mathrm{df}=2, \mathrm{p}$-value $=3.197 \mathrm{e}-10$ 
Os valores apresentados na Tabela 1 revelam que a porcentagem de escore com valor 1 e a média dos escores de repetição aumentam à medida que aumenta a faixa etária, enquanto a porcentagem de escores com valores menores que 0,70 diminuem. Esses resultados demostram que a acurácia no teste de repetição é maior entre as crianças mais velhas. Além disso, as médias obtidas para os escores de cada grupo apesentam valores próximos de um, sendo a média mais alta a do grupo etário de 9 a 12 anos. O resultado do p-valor mostra que essas diferenças são significativas.

Os Gráficos 1 e 2, a seguir, apresentam os escores obtidos para as duas variáveis controladas no teste de repetição de não-palavras, tamanho e frequência das sílabas constituintes da não-palavra. A análise do teste Kruskal-Wallis revelou valores estatisticamente significativos tanto para a frequência das sílabas que compõem os estímulos como para o tamanho da não-palavra, com p-valores de 1.606e-09 e 0.001392, respectivamente.

Os valores de acurácia de repetição das não-palavras em função da frequência estão representados no Gráfico esquemático 1.

GRÁFICO 1 - Distribuição dos escores de acurácia de repetição em função da frequência das sílabas dos estímulos

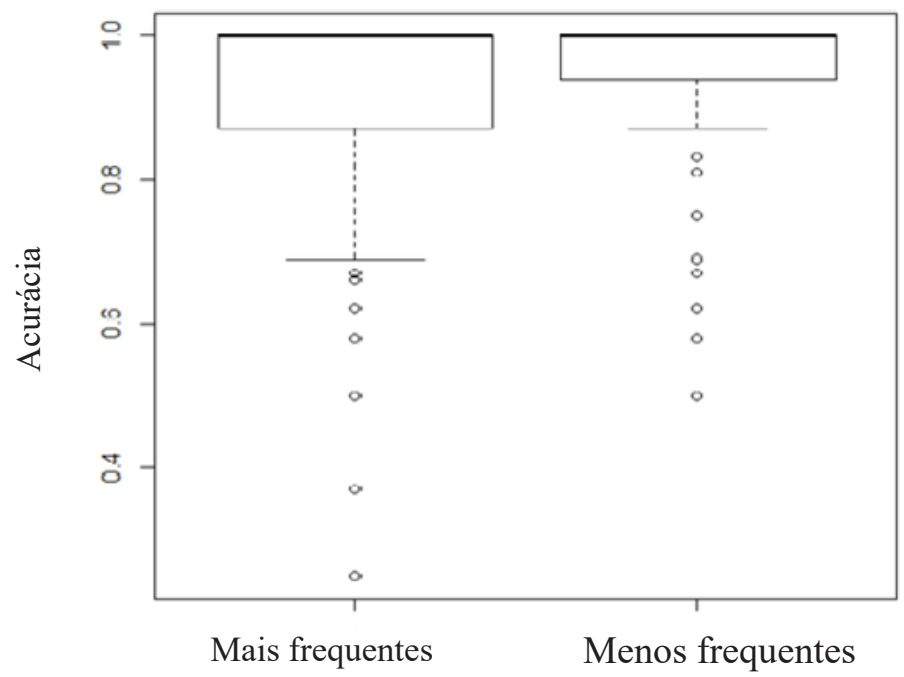

kruskal-wallis chi-squared $=36.4012, \mathrm{df}=1, \mathrm{p}$-value $=1.606 \mathrm{e}-09$ 
No Gráfico 1, observa-se que há uma concentração de respostas próximas de 1 , com a mediana se sobrepondo à linha dos valores máximos, caracterizando assim que mais de $50 \%$ dos índices tanto de não-palavras compostas por sequências fonotáticas de alta frequência como as de baixa frequência estão com escores em 1 ou muito próximo de 1 . No entanto, há valores fora desse intervalo, muitos considerados discrepantes, como demonstram os círculos fora das caixas. ${ }^{5}$ Para as nãopalavras com sílabas menos frequentes, há escores mais discrepantes, de menor acurácia, do que para as não-palavras com sílabas mais frequentes.

O Gráfico Esquemático 2, a seguir, apresenta a distribuição da acurácia de repetição em relação ao tamanho da não-palavra.

GRÁFICO 2 - Distribuição dos escores de acurácia de repetição em função do tamanho da não-palavra

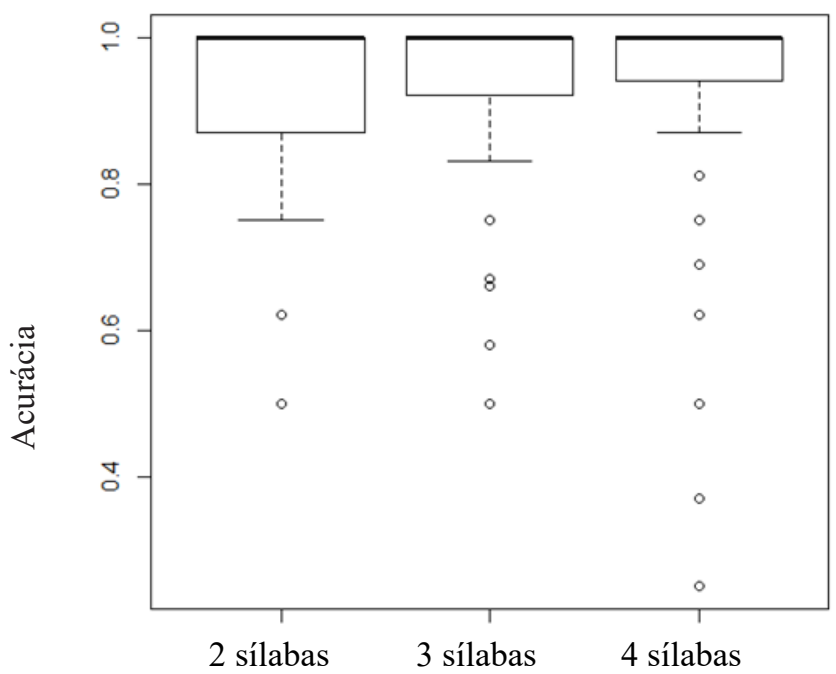

kruskal-wallis chi-squared $=13.1547, \mathrm{df}=2, \mathrm{p}$-value $=0.001392$

\footnotetext{
${ }^{5}$ A medida utilizada é a divisão em quartis. Essa medida divide o conjunto de dados em quatro partes iguais. Os valores menores estão baixo que o valor da cerca interior, isto é, Q1, CI=Q1-1,5(Q3-Q1), em que Qi são os quartis (GOMES; MENDES; GOMES., 2015).
} 
Observa-se que há uma concentração de respostas próximas de 1, com a mediana sobrepondo-se à linha dos valores máximos, caracterizando também que mais de 50\% dos escores para as não-palavras de 2, 3 e 4 sílabas estão com escores em 1 ou muito próximos de 1 . No entanto, há valores fora desse intervalo, como demonstram os círculos fora das caixas. Há mais quantidade de respostas com escores baixos para as não-palavras de 4 sílabas. Os escores das não-palavras de 2 e 3 sílabas apresentam índices dentro do mesmo intervalo.

Os escores obtidos no teste de repetição de não-palavras em função do tamanho do léxico estão representados no Gráfico 3 a seguir. Com relação ao grau de acurácia de repetição de não-palavra em função do tamanho do léxico, o teste de coeficiente da reta (chi-squared= 0.0395 , f-statistics $(\mathrm{df}=1,1970)=81.0406 \mathrm{p}$-value $=0)$ mostra que há uma inclinação $(\neq 0)$, o que permite verificar uma relação entre o grau de acurácia e o tamanho do léxico.

GRÁFICO 3 - Diagrama de dispersão dos escores de acurácia de repetição de não-palavras em função do tamanho do léxico

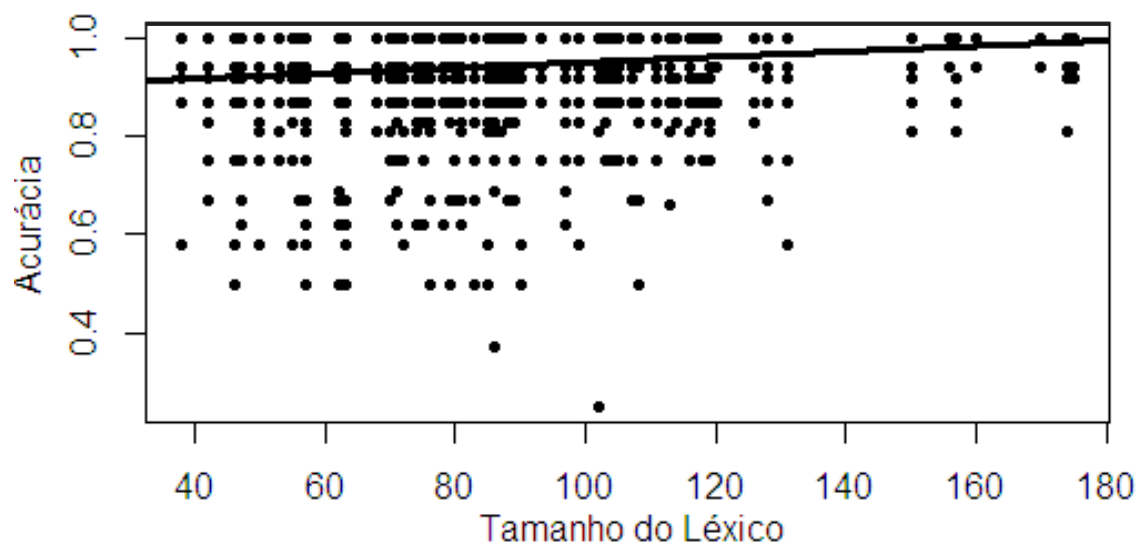

De acordo com o que se observa no Gráfico 3, existem escores de repetição altos com léxico de tamanho menor e escores de repetição altos com tamanho de léxico maior. Entretanto, pode-se verificar que, a partir de um determinado tamanho do léxico, em torno dos 110 pontos, começam a escassear os escores baixos e, em tamanho de léxico a partir dos 150 pontos, os escores baixos desaparecem. 
A fim de se obterem informações mais detalhadas a respeito da relação entre grau de acurácia e tamanho do léxico, os escores relativos a estímulos constituídos por sílabas de alta e baixa frequência foram separados e representados nos dois gráficos de dispersão a seguir e foram estabelecidas as retas de tendência para os dois tipos de estímulos, conforme pode ser observado nos Gráficos 4 e 5. Como verificado em Gomes, Mendes e Gomes (2015), em um modelo de regressão com variáveis indicadoras, os resultados para os coeficientes das retas mostraram que há inclinação. Portanto, os valores do eixo y, escore de acurácia, têm relação de dependência com os do eixo $\mathrm{x}$, tamanho do léxico $(\mathrm{R} 2=0.9912$, p-valor $=0.000)$.

GRÁFICO 4 - Grau de acurácia

na repetição de estímulos

de baixa frequência

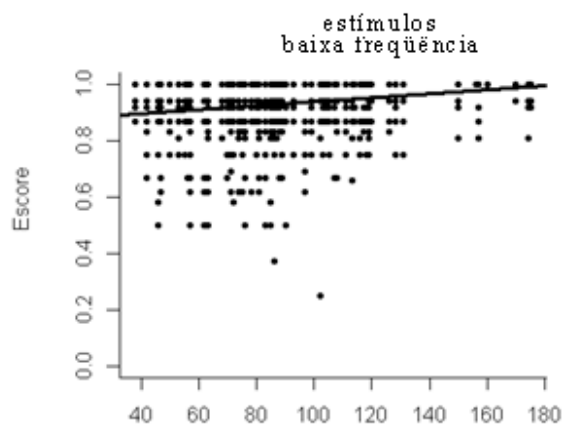

GRÁFICO 5 - Grau de acurácia na repetição de estímulos de alta frequência

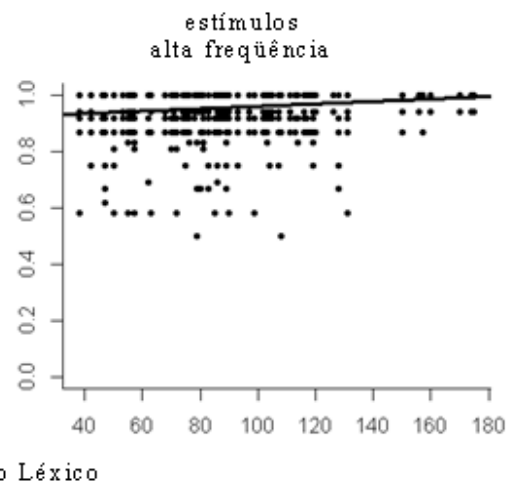

Em ambos os gráficos, observa-se que os escores de acurácia mais baixos, abaixo de 0,80 , foram obtidos no desempenho de crianças com o léxico de tamanho menor e que há mais escores de acurácia mais baixos para estímulos constituídos por sílabas menos frequentes. As curvas de tendências no Gráfico 4 e no Gráfico 5 indicam que o efeito da frequência está presente quando o tamanho do léxico é menor para os dois tipos de estímulos.

As análises estatísticas revelaram, conforme descrito nos resultados, que a idade, o tamanho dos estímulos, a frequência das sílabas constituintes das não-palavras e o tamanho do léxico apresentaram 
relevância significativa em relação ao desempenho na repetição das nãopalavras pelas crianças. Os resultados para os grupos etários revelaram que a acurácia na repetição de não-palavras melhora com o aumento da idade. Esses achados estão dentro do esperado, visto que o desenvolvimento fonológico se estabelece de maneira gradual, e, portanto, as crianças mais novas estão em uma etapa desse desenvolvimento anterior ao das crianças mais velhas.

A relação entre a variável frequência de tipo e o grau de acurácia na repetição revelou que os estímulos formados de sílabas frequentes foram repetidos de forma mais acurada que os estímulos com constituintes menos frequentes. Esses resultados estão de acordo com os resultados obtidos por Gathercole (1995), Munson et al. (2005) e Beckman et al. (2007), em trabalhos com crianças com desenvolvimento típico e atípico, conforme mencionado anteriormente nas seções 2 e 3 .

A relação entre a variável tamanho do estímulo e o grau de acurácia demonstrou que a repetição de não-palavras de 2 e 3 sílabas apresentou uma melhor acurácia do que a de não-palavras de 4 sílabas. A explicação para a presença desse efeito pode estar ligada a questões de memória de trabalho, visto que itens mais longos demandam mais esforços para serem retidos e lembrados, e consequentemente, podem gerar impactos no desempenho de repetição. Também o trabalho de Frisch et al. (2000) encontrou efeito de tamanho do estímulo no processamento de não-palavras por falantes adultos.

Com relação ao tamanho do léxico, foi observado que o aumento da acurácia está relacionado ao aumento do vocabulário dos participantes. Apesar de haver escores altos de acurácia com índices mais baixos de tamanho de léxico, os escores baixos tendem a desaparecer à medida que o léxico aumenta. Esses resultados são também compatíveis com os achados de Munson, Kurtz et al. (2005) e Beckman et al. (2007) realizados com crianças de população clínica e com crianças com desenvolvimento típico.

Os resultados expostos na literatura somados aos encontrados nesta pesquisa, referentes à relação entre tamanho do léxico e grau de acurácia na repetição de não-palavras, indicam que o léxico confere robustez às abstrações da gramática fonológica necessárias para 0 processamento das não-palavras. Esses resultados fornecem evidências para a hipótese inicial de que o conhecimento fonológico emerge das representações das formas das palavras no léxico. Assim, quanto maior 
o léxico mais abstrações farão parte da gramática abstraída pelo falante, e, consequentemente, melhor a acurácia na repetição das não-palavras.

A fim de melhor investigar a hipótese entre a relação do efeito de frequência e tamanho do léxico, os escores obtidos para os diferentes estímulos foram separados em função da frequência e observados em relação ao tamanho do léxico. As retas de tendências das não-palavras frequentes e não frequentes revelaram, através de suas inclinações, que tanto a repetição de não-palavras compostas por sílabas de alta frequência como as não-palavras compostas por sílabas de baixa frequência são influenciadas pelo tamanho do léxico, visto que, à medida que o léxico aumenta, aumentam também os escores de repetição de não-palavras.

Conforme mencionado na seção 3 , existe uma discussão na literatura a respeito de se o processamento das não-palavras com componentes de baixa frequência é mediado pelo conhecimento lexical ou pela memória de curto prazo. Gathercole $(1995,2006)$ defende que o processamento desses estímulos é baseado nas informações fonológicas retidas na memória de curto prazo, já que o indivíduo não possui essa informação na sua memória de longo prazo, ao passo que as nãopalavras com sequências fonotáticas de alta frequência são mediadas pelo conhecimento lexical de longo termo. Outros autores, como Frisch, Large e Pisoni (2000) e Munson, Kurtz e Windsor (2005), defendem que as não-palavras compostas por sequências de alta e de baixa frequência são processadas a partir de inferências probabilísticas sobre os padrões abstraídos do léxico. Entretanto, os resultados desta pesquisa não fornecem evidência para a discussão do papel da memória de curto prazo na repetição de não-palavras com constituintes de baixa frequência, embora indiquem que o léxico exerce um papel nesse tipo de tarefa para este tipo de estímulo. Esteves (2013), em trabalho com populações clínicas de crianças com diagnóstico de DEL, distúrbio fonológico e dislexia concluiu que o tamanho do léxico exerce uma função maior no processamento das não-palavras que a memória de curto prazo.

Além disso, os resultados deste estudo, de acordo com o que pode ser observado nos Gráficos 3, 4 e 5, também permitiram verificar que as crianças com um tamanho de léxico em torno de 150, que corresponde ao tamanho de léxico esperado para crianças com 13 anos de idade, não são sensíveis ao efeito de frequência para o tipo de estímulo usado, uma vez que os escores obtidos se concentram em torno de 0,80 e 1 . Ou seja, esse tamanho de léxico parece ser suficiente para dar robustez a 
sílabas CV do português independentemente da sua frequência de tipo. $\mathrm{O}$ efeito detectado da frequência de tipo é indicativo de que as crianças ainda não atingirem um tamanho de léxico capaz de conferir robustez às representações mapeadas nos moldes lexicais dos estímulos do teste.

\section{Considerações Finais}

Os resultados encontrados nesta pesquisa ratificam as hipóteses sobre o conhecimento fonológico do falante de acordo com os Modelos Baseados no Uso, segundo as quais o conhecimento fonológico abstrato emerge das palavras no léxico e que o falante é sensível às informações probabilísticas (frequência de tipo) que emergem dessas representações. Assim, no processo de aquisição da linguagem, sílabas ou relações fonotáticas mais frequentes tendem a ser mais robustamente representadas na gramática fonológica à medida que a aquisição lexical progride, e isso influencia a acurácia em testes de repetição. No entanto, o efeito de frequência tende a desaparecer à medida que o léxico aumenta e alcança um determinado tamanho, ou seja, é de tamanho suficiente para garantir robustez a qualquer estrutura fonológica da língua, independentemente de sua frequência.

Os achados desta pesquisa também contribuem para a prática fonoaudiológica porque fornecem informações sobre a aquisição do conhecimento fonológico abstrato referente às relações fonotáticas e moldes lexicais (templates) e sua relação com o tamanho do léxico em crianças com desenvolvimento típico, que servem de base para a avaliação e terapêutica de crianças com desenvolvimento atípico, e apontam a importância dessa estimulação no processo terapêutico.

\section{Referências}

ARAÚJO, M. V. M.; MARTELTO, M. R. F.; SCHOEN-FERREIRA, T. H. Avaliação do vocabulário receptivo de crianças pré-escolares. Estudos de Psicologia, Campinas, v. 27, p.169-176, 2010. https://doi.org/10.1590/ s0103-166x2010000200004.

BECKMAN, M. E.; MUNSON, B.; EDWARDS, J. The influence of vocabulary growth on developmental changes in types of phonological knowledge. In: COLE, J.; HUALDE, J. (Ed.). Papers in Laboratory Phonology 9. New York: Mouton de Gruyter, 2007. p. 241-264. 
BECKNER, C. et al. Language is a complex adaptative system: position paper. Language Learning, Michigan, v. 59, n. 1, p. 1-26, 2009.

BYBEE, J. Regular morphology and the lexicon. Language and Cognitive Processes, Routledge, v. 10, p.: 425-455, 1995. https://doi. org/10.1080/01690969508407111.

BYBEE, J. Phonology and language use. New York: Cambridge University Press, 2001. v. 23, p. 30-87. https://doi.org/10.1017/ CBO9780511612886.

BYBEE, J. Language, usage and cognition. Cambridge: Cambridge University Press, 2010. https://doi.org/10.1017/CBO9780511750526.

BYBEE, J. Language Change. Cambridge: Cambridge University Press, 2015.

CAPOVILLA, A. G. S.; CAPOVILLA, F. C. Desenvolvimento lingüístico na criança brasileira dos dois aos seis anos: tradução e estandartização do Peabody Picture Vocabulary Test de Dunn \& Dunn e Language Development Survey de Rescorla. Ciência Cognitiva: Teoria, Pesquisa e Aplicação, v. 1, p. 353-380, 1997.

CLOPPER, C. G.; CONREY, B. L.; PISONI, D. B. Effects of talker gender on dialect categorization. Journal of Language and Social Psychology, Santa Barbara, CA, v. 24, p. 182-206, 2005. https://doi. org/10.1177/0261927x05275741.

COLEMAN, J.; PIERREHUMBERT, P. Stochastic Phonological Grammars and acceptability. In: MEETING OF THE ACL SPECIAL INTEREST GROUP IN COMPUTATIONAL PHONOLOGY, $3^{\text {rd }}$, 1997, Somerset, NJ.: Proceedings... Somerset, NJ: Association for Computational Linguistics, 1997. p. 49-56.

DOCHERTY, G.; FOULKES, P. Speaker, speech and knowlwdge of sounds. In: BURTON-ROBERTS, N.; CARR, P.; DOCHERTY, G. (Ed.). Phonological Knowledge: conceptual and empirical issues. Oxford: Oxford University Press, 2000. p.105-129. https://doi.org/10.1016/j. lingua.2013.01.011.

DOCHERTY, G.; FOULKES, P. An evaluation of usage-based approaches to the modelling of sociophonetic variablity. Lingua, Elsevier, v. 142, p. 42-56, 2014. 
DUNN, L. M.; DUNN, L. M. Peabody Picture Vocabulary Test $-3^{\text {th }}$ Edition (Ppvt-III). Minnesota: American Guidance Service, 1997.

ESTEVES, C. O Conhecimento Fonológico de Crianças com Dislexia, Desvio Fonológico e Distúrbio Específico de Linguagem: Uma Análise Multirrepresentacional da Linguagem. 2013, $131 \mathrm{f}$. Tese (Doutorado em Linguística) - Faculdade de Letras da Universidade Federal do Rio de Janeiro, 2013.

FERRACINI, F.; CAPOVILLA, A. G. S.; DIAS, N. M.; CAPOVILLA, F. C. Avaliação de vocabulário expressivo e receptivo na educação infantil. Revista de Psicopedagogia, São Paulo, v. 23, n. 206, p. 124-133, 2006.

FERGUSON, C. A.; FARWELL, C. Words and sounds in early language acquisition. Language, The Linguistic Society of American, v. 51, p. 419-439, 1975. https://doi.org/10.2307/412864.

FORSTER, K. I.; FORSTER, J. C. DMDX: A Windows Display Program with Millisecond Accuracy. Behavior Research Methods, Instruments, \& Computers, Interaction Design Foudation, v. 35, p. 116-124, 2003.

FRISCH, S.; LARGE, N.; PISONI D. Perception of wordlikeness: effects of segment probability and length on the processing of nonwords. Journal of Memory and Language, Elsevier, v. 42, p. 481- 496, 2000. https://doi. org/10.1006/jmla.1999.2692.

GATHERCOLE, S. Is nonword repetition a test of phonological memory or long-term knowledge? It all depends on nonwords. Memory \& Cognition, Springer International Publishing AG, v. 23, n. 1, p. 83-94, 1995.

GATHERCOLE, S.E. Nonword repetition and word learning: the nature of the relationship. Applied Psycholinguistics, Cambridge, v. 27, p. 513-554, 2006. https://doi.org/10.1017/s0142716406060383.

GOLDINGER, S. D. Words and voices: episodic traces in spoken word identification and recognition memory. Journal of Experimental Psychology: Learning, Memory, and Cognition, Washington, v. 22, p. 1166-1183, 1996. 
GOMES, C. A.; MENDES, S. C.; ESTEVES, C. O.; SILVA, M. B.; GOMES, G. C. Efeito de wordlikeness no processamento de não-palavras por falantes do português brasileiro. Revista de Estudos da Linguagem, Belo Horizonte, v. 23, p. 195-210, 2015. https://doi.org/10.17851/22372083.23.1.195-210.

GOMES, G. C.; MENDES, S. C.; GOMES, C. A. Um modelo em análise de regressão para avaliar o conhecimento fonológico de crianças com desenvolvimento típico. In: SIMPÓSIO BRASILEIRO DE PESQUISA OPERACIONAL, XLVII., 2015. Porto de Galinhas-PE, 2015.

HAZAN, V.; BARRETT, S. The development of phonemic categorization in children aged 6-12. Journal of Phonetics, Elsevier, v. 28, p. 377-396, 2000. https://doi.org/10.1006/jpho.2000.0121.

HINTZMAN, D. L. "Schema abstraction" in a multiple-trace memory model. Psychological Review, Washington, v. 93, p.411-428, 1986. https://doi.org/10.1037/0033-295x.93.4.411.

INGRAM, D. The measurement of whole-word productions. Journal of Child Language, Cambridge, v. 29, p. 713-733, 2002. https://doi. org/10.1017/s0305000902005275.

JOHNSON, K. Speech perception without speaker normalization: an exemplar model. In: JOHNSON, K.; MULLENNIX, J. W. (Ed.). Talker variability in speech processing. San Diego: Academic Press, 1997. p. 145-166.

MENDES, S. do C. Habilidades do processamento auditivo e conhecimento fonológico em crianças com desenvolvimento típico. 2014. 144f. Dissertação (Mestrado em Linguística) - Faculdade de Letras Universidade Federal do Rio de Janeiro, 2014.

MUNSON, B.; EDWARDS, J.; BECKMAN, M. E. Phonological Knowledge in Typical and Atypical Speech-Sound Development. Topics of Language Disorders, American Speech Language-Hearing Association, v. 25, n. 3, p.190-206, 2005.

MUNSON, B.; KURTZ, B. A.; WINDSOR, J. The influence of vocabulary size, phonotactic probability, and wordlikeness on nonword repetitions of children with and without specific language impairment. Journal of Speech, Language, and Hearing Research, American Speech-LanguageHearing Association, v. 48, p. 1033-1047, 2005. 
NOSOFSKY, R. M. Attention, similarity, and the identificationcategorization relationship. Journal of Experimental Psychology: General, Washington, v. 115, p.39-57, 1986. https://doi.org/10.1037/00963445.115.1.39.

PELUCCHI, B; HAY, J. F.; SAFFRAN, J. R. Statistical learning in a natural language by 8 -month-old infants. Child Development, Child Development Perspectives, Monographs of The Society for Research in Child Developement, v. 80, p. 674-685, 2009.

PIERREHUMBERT, J. Knowledge of variation. In: MEETING OF THE CHICAGO LINGUISTIC SOCIETY, 30 ${ }^{\text {th }}, 1994$, Chicago. Knowledge of Variation. Papers from the Parasession on Variation. Chicago: Chicago Linguistic Society, 1994. 25 p.

PIERREHUMBERT, J. What people know about sounds of language. Studies in the Linguistic Sciences, University of Illinois, v.29, p.111-120, 1999.

PIERREHUMBERT, J. Exemplar dynamics: word frequency, lenition, and contrast. In: BYBEE, J.; HOPPER, P. (Ed.). Frequency effects and the emergence of lexical structure. Amsterdam: John Benjamins, 2001. p. 137-157.

PIERREHUMBERT, J. Phonology: discrimination and robustness. In: BOD, N. R.; HAY, J.; JANNEDY, S. (Ed.). Probability theory in linguistics. Cambridge: MIT Press, 2003. p. 177-228

PIERREHUMBERT, J. The dynamic lexicon. In: COHN, A.; HUFFMAN, M.; FOUGERON, C. (Ed.). Handbook of Laboratory Phonology. Oxford: Oxford University Press, 2012. p. 173-183.

PISONI, D. B. et al. Speech perception, word recognition and the structure of the lexicon. Speech Communication, Bethesda, MD, USA, v. 4, p.75-95, 1985. https://doi.org/10.1016/0167-6393(85)90037-8.

SAFFRAN, J. R; ASLIN, R. N.; NEWPORT, E. L. Statistical learning by 8-month-old infants. Science, Bethesda, MD, USA, v. 274, p.19261928, 1996.

SILVA, T. C.; GOMES, C. A. Representações múltiplas e organização do componente linguístico. Forum Linguístico, Florianópolis, v. 4, n. 1, p. 147-177, 2007. 
THIESSEN, E. D; SAFFRAN, J. R. When cues collide: use of stress and statistical cues to word boundaries by 7- to 9-month-old infants. Developmental Psychology, Washington, v. 39, p. 706-716, 2003. https:// doi.org/10.1037/0012-1649.39.4.706.

VELLEMAN, S. L.; VIHMAN, M. M. Phonological development in infancy and early childhood: Implications for theories of language learning. In: PENNINGTON, M. C. (Ed.). Phonology in Context. Luton: Macmillan, 2006. p. 25-50.

VIHMAN, M. M. Word learning and the origins of phonological system. In: FOSTER-COHEN, S. (Ed.). Advances in language acquisition. Luton: Macmillan, 2009. p. 15-39. https://doi.org/10.1057/9780230240780_2.

VIHMAN, M. M. Phonological development: the first two years. $2^{\text {nd }}$ ed. Malden, MA: Wiley-Blackwell, 2014.

VIHMAN, M. M.; KUNNARI, S. The sources of phonological knowledge: a cross-linguistic perspective. Recherches Linguistiques de Vincennes, Saint-Denis, v. 35, p. 133-164, 2006.

VIHMAN, M. M.; CROFT, W. Phonological development: toward a 'radical' templatic phonology. Linguistics, The University of York, v. 45, p. 683-725, 2007. https://doi.org/10.1515/ling.2007.021.

VITEVITCH, M. S.; LUCE, P. A. Probabilistic phonotactics and neighborhood activation in spoken word recognition. Journal of Memory and Language, Elsevier, v. 40, p.374-408, 1999. https://doi.org/10.1006/ jmla.1998.2618. 
